\title{
Search, Money and Capital: A Neoclassical Dichotomy*
}

\author{
S. Borağan Aruoba \\ Department of Economics \\ University of Pennsylvania \\ aruoba@econ.upenn.edu
}

\author{
Randall Wright \\ Department of Economics \\ University of Pennsylvania \\ rwright@econ.upenn.edu
}

September 9, 2002

\begin{abstract}
Recent work has reduced the gap between search-based monetary theory and mainstream macroeconomics by incorporating into the search model some centralized markets as well as some decentralized markets where money is essential. This paper takes a further step towards this integration by introducing labor, capital and neoclassical firms. The resulting framework nests the search-theoretic monetary model and a standard neoclassical growth model as special cases. Perhaps surprisingly, it also exhibits a dichotomy: one can determine the equilibrium path for the value of money independently of the paths of consumption, investment and employment in the centralized market.
\end{abstract}

${ }^{*}$ We thank K. Burdett, G. Eudey, R. Lagos, M. Molico and C. Waller for their input, as well as the NSF and the Cleveland Fed for research support. 


\section{Introduction}

There seems to be a big distance between standard macroeconomics and the branch of monetary theory with explicit microfoundations based on search, or matching, theory. As Azariadis (1993) put it, "Capturing the transactions motive for holding money balances in a compact and logically appealing manner has turned out to be an enormously complicated task. Logically coherent models such as those proposed by Diamond (1982) and Kiyotaki and Wright (1989) tend to be so removed from neoclassical growth theory as to seriously hinder the job of integrating rigorous monetary theory with the rest of macroeconomics." As Kiyotaki and Moore (2001) more recently put it, "The matching models are without doubt ingenious and beautiful. But it is quite hard to integrate them with the rest of macroeconomic theory - not least because they jettison the basic tool of our trade, competitive markets."

Recent work has gone some distance towards closing the gap between the search-based approach and mainstream macroeconomics. An example is the model in Lagos and Wright (2002a), hereafter referred to as LW. The innovation in LW is to bring competitive markets back on board in a way that maintains an essential role for money and at the same time greatly increases the tractability of the search framework. In the LW environment there is decentralized trade in anonymous markets with bilateral random matching, as in a typical search model, but after each round of decentralized trade a centralized market convenes. In the centralized market agents not only produce and exchange goods for consumption purposes, they also trade to adjust their money balances, which may have changed from the desired level during the previous round of decentralized trade. Under the assumption that utility is quasi-linear in one of the goods traded in the centralized market, it turns out that all agents adjust to the same money balances. Hence, at the start of every period there will be a degenerate distribution of money holdings. ${ }^{1}$

\footnotetext{
${ }^{1}$ See Lagos and Wright (2002b), Rocheteau and Wright (2002), Berentsen, Lagos and Rocheteau (2002)
} 
This resolves a complicated technical problem - solving for and keeping track of the money distribution - which often forced people to make undesirably strong assumptions in earlier search-based models, like severe restrictions on how much money agents can hold (typically it was restricted to 0 or 1 unit). The LW framework allows one to address many issues for which models with these severe restrictions are ill-suited, and yet it is very simple. The simplicity comes at a cost since, after all, having an endogenous non-degenerate distribution of money holdings may be interesting and relevant for some questions. ${ }^{2}$ Presumably, however, there are some interesting questions in monetary economics for which an endogenous non-degenerate distribution is not critical. For such questions, the LW framework provides a tractable model with explicit microfoundations, and no restrictions on money holdings, which means that it can be more easily used to discuss monetary policy and other issues that were difficult in earlier search models.

This is the sense in which we mean recent work has gone some distance towards integrating search-based models theory and mainstream macroeconomics. The point of the current paper is to show that with a little effort one can take a much bigger step towards this integration. Existing versions of the LW framework still do not look much like the neoclassical growth model. Indeed, not much happens in the centralized market in these models, and it is there mainly to render the distribution of money holdings in the decentralized market degenerate. Yet once this centralized market is up and running, one can do a lot more. Here we introduce labor, capital, and a neoclassical production function. The result integrates a

and Berentsen, Rocheteau and Waller (2002) for extensions and applications of the basic framework. A related but also quite different approach, dating back to Shi (1997), uses the assumption of large families rather than competitive markets to render the money distribution degenerate. In Shi (1999) and also Faig (2001), these families produce specialized goods that they can either trade or keep within the household to be used as capital. Here we will also introduce capital, but as a general good that is traded on a centralized market, much more in the spirit of standard macroeconomics.

${ }^{2}$ See Molico (1999) for an example where a non-degenerate distribution is interesting; see Wallace (2002) for a general discussion. The standard references for models that assume $m \in\{0,1\}$, so as to avoid dealing with this distribution, include Kiyotaki and Wright (1993), Shi (1995) and Trejos and Wright (1995). 
standard growth model and the search-theoretic monetary model; indeed, these two models emerge as special cases.

Perhaps surprisingly, when we specify the model in what we think of as a very natural way, an interesting dichotomy emerges: it is possible to partition the equilibrium conditions in such a way that one can solve independently for the allocation in the centralized and decentralized markets. The nominal price level ties these markets together, since money is traded in both markets, but it turns out that although the price level affects the allocation in the decentralized market in an important way, in the centralized market it does not affect aggregate activity or welfare. Many policy implications follow from this result. For example, a change in the rate of monetary expansion can affect the inflation rate and hence the price level, and this affects consumption in the decentralized market, but is completely neutral in terms of the aggregate labor market or capital accumulation. ${ }^{3}$

These policy implications ought to be interpreted cautiously. First, the fact that inflation has no impact on the aggregate labor market or capital accumulation does not mean that inflation does not matter, since it does affect economic activity in the decentralized market and hence welfare. Second, the dichotomy and its implied policy implications of course depend crucially on certain assumptions. So, while our model does integrate neoclassical growth theory and monetary models with explicit microfoundations in a simple and natural way, we think of it mainly as a benchmark from which policy discussions can proceed. Thus, it may or may not be that monetary policy has real effects on centralized markets in actual

\footnotetext{
${ }^{3}$ Our result is different from the classical dichotomy. As Sargent (1979) put it, "A macroeconomic model is said to dichotomize if a subset of equations can determine the values of all real variables with the level of the money supply playing no role in determining the equilibrium value of any real variable. Given the equilibrium values of the real variables, the level of the money supply helps determine the equilibrium values of all nominal variables that are endogenous but cannot influence any real variable. In a system that dichotomizes the equilibrium values of all real variables are independent of the absolute price level." This is not quite the case here, since the amount of output that one gets for a dollar in decentralized trading (a real variable) does depend on the absolute price level and hence on monetary policy - but the real variables from the centralized market (employment, consumption and investment) are independent of the price level and monetary policy.
} 
economies, but if so, a reasonable model of this will have to do something different from what we do here. ${ }^{4}$

The rest of the paper is organized as follows. Section 2 presents the basic structure by reviewing the LW model. Section 3 shows how to introduce capital accumulation. Section 4 adds labor as well as capital. Section 5 endogenizes search intensity, or shopping time. All of these models display the strong dichotomy referred to above. Section 6 concludes.

\section{The Basic Model}

Time is discrete and continues forever. There is a $[0,1]$ continuum of infinitely-lived agents. There are two types of commodities: a general good, and a set of special goods. All goods are nonstorable and perfectly divisible. All agents consume the general good, but each agent derives utility from only some subset of special goods. All agents can produce the general good, but each has a technology that allows him to produce only one special good. No agent consumes the special good he produces. For a random pair of agents, we assume the following: with probability $\Delta$ both like the special good the other can produce (called a double coincidence); with probability $\sigma$ one likes the other's good but not vice-versa (called a single coincidence); and with probability $1-\Delta-2 \sigma$ neither likes the other's good, where $\Delta \geq 0, \sigma>0$ and $\Delta+2 \sigma \leq 1$

In addition to consumption goods, there is another object called money that cannot be consumed or produced by any private agent. Money is perfectly divisible and storable, and agents can carry any non-negative quantity of money. Let $F_{t}(m)$ denote the CDF of money holdings across agents, where $\int m_{t} d F_{t}\left(m_{t}\right)=M_{t}$ is the total amount of money, at date $t$. The money supply changes over time according to $M_{t+1}=\left(1+\tau_{t}\right) M_{t}$, where the growth rate $\tau_{t}$ need not be constant. New money is injected in the form of lump-sum transfers, or

\footnotetext{
${ }^{4}$ By analogy, in the basic growth model, the welfare theorems hold, which obviously also entails some stark conclusions about policy, yet this model serves us well as a benchmark for policy discussions.
} 
taxes if $\tau_{t}<0$. To be precise, we assume each period is divided in two subperiods - say, day and night - and money transfers occur at the end of the second subperiod. Agents discount between periods at rate $\beta$, but not between day and night within a period (this is without loss in generality).

During the day (i.e. in the first subperiod), agents participate in a decentralized market with bilateral random matching. The probability of meeting anyone is $\alpha$ and each meeting is a random draw from the population. These meetings are anonymous, which prevents agents from trading any promises to be fulfilled in the future or even later that same period (Kocherlakota [1998]; Wallace [2001, 2002]). Also, during the day agents can produce special goods but not general goods. By contrast, during the night agents can produce general but not special goods, and they participate in a centralized market. Given this environment, the feasible trades are as follows: special goods can be traded for other special goods or for money during the day; and general goods can be traded for money at night.

In any single coincidence meeting in the decentralized market, we call the agent that likes that other's good the buyer, and the other agent the seller. In such a meeting let $q_{t}(m, \tilde{m})$ be the amount of goods and $d_{t}(m, \tilde{m})$ the amount of money they exchange, where $m$ is the money holdings of the buyer and $\tilde{m}$ is the money holdings of the seller. Also, let $B_{t}(m, \tilde{m})$ be the payoff from a trade in a double coincidence meeting when the agents hold $m$ and $\tilde{m}$. These variables will be determined by bargaining. By contrast, in the centralized market that convenes at night agents behave competitively - i.e., they trade general goods and money taking prices parametrically. We normalize the price of a general good in the night market to 1 and let $\phi_{t}$ be the amount of general goods that a dollar will buy; thus $p_{t}^{g}=1 / \phi_{t}$ is the nominal price of general goods at $t$.

The utility of consuming $q$ units of a special good that one likes is $u(q)$, and the cost of producing $q$ units of a special good is $c(q)$. Assume $u$ and $c$ are $\mathbb{C}^{n}(n$ times continuously 
differentiable) with $n \geq 3$, where $u^{\prime}>0, c^{\prime}>0, u^{\prime \prime}<0$ and $c^{\prime \prime} \geq 0$. Also, $u(0)=c(0)=0$ and $u(\bar{q})=c(\bar{q})$ for some $\bar{q}>0$. For certain results we need an assumption on $u^{\prime \prime \prime}$ which is conveniently stated by saying that marginal utility is log-concave (i.e., the log of $u^{\prime}$ is concave). Let $q^{*}$ denote the efficient quantity, which solves $u^{\prime}\left(q^{*}\right)=c^{\prime}\left(q^{*}\right) ; q^{*}$ is the amount agents would agree ex ante that they should trade in each decentralized meeting if they could commit to such an arrangement - but of course, they cannot so commit, since if they could money would be inessential. For general goods, $U$ and $C$ are the utility of consumption and cost of production. Assume $U$ and $C$ are $\mathbb{C}^{n}$ with $n \geq 2$, where $U^{\prime}>0, C^{\prime}>0, U^{\prime \prime} \leq 0$, $C^{\prime \prime} \geq 0$, and $U^{\prime}\left(x^{*}\right)=C^{\prime}\left(x^{*}\right)$ for some $x^{*}>0$ with $U\left(x^{*}\right)>C\left(x^{*}\right)$. We need either $U$ or $C$ to be linear; for now we take $C(y)=y .^{5}$

Let $W(s)$ be the value function of an agent entering the night market and $V(s)$ the value function of an agent entering the day market with individual state variable $s$. For now, one's state is simply one's money holdings, $s=m$, but we introduce this notation since $s$ will include other objects in the models analyzed below. The aggregate state is the distribution $F$, which will remain implicit in the functional notation. Bellman's equation is

$$
\begin{aligned}
V_{t}(m) & =\alpha \sigma \int\left\{u\left[q_{t}(m, \tilde{m})\right]+W_{t}\left[m-d_{t}(m, \tilde{m})\right]\right\} d F_{t}(\tilde{m}) \\
& +\alpha \sigma \int\left\{-c\left[q_{t}(\tilde{m}, m)\right]+W_{t}\left[m+d_{t}(\tilde{m}, m)\right]\right\} d F_{t}(\tilde{m}) \\
& +\alpha \Delta \int B_{t}(m, \tilde{m}) d F_{t}(\tilde{m})+(1-2 \alpha \sigma-\alpha \Delta) W_{t}(m) .
\end{aligned}
$$

The first term is the expected gain from buying in a single-coincidence meeting; the second is the expected gain from selling in a single-coincidence meeting; the third is the expected gain from a double-coincidence meeting; and the last term is the expected value of not trading in the day market and going to the centralized market with $m$. We are not restricting anything

\footnotetext{
${ }^{5}$ Below we show the case where $U$ is linear and $C$ strictly convex is basically identical. Once we introduce a neoclassical production function we assume utiltiy is linear in lesiure. The reason we need linearity somewhere in preferences over general goods is to eliminate wealth effects, because then all agents will take the same amount of money out of the centralized market, regardless of their histories. If this were not the case, the model would still be well-specified, but it would be much less tractable.
} 
to be stationary here, although we sometimes drop the subscript $t$ when there is no risk of confusion.

The problem of an agent in the centralized market is

$$
\begin{aligned}
W(m) & =\max _{x, y, m^{\prime}} U(x)-y+\beta V\left(m^{\prime}+\tau M\right) \\
\text { s.t. } x & =\phi\left(m-m^{\prime}\right)+y .
\end{aligned}
$$

Thus, he chooses general good consumption and production, $x_{t}$ and $y_{t}$, and takes $m_{t+1}=$ $m_{t}^{\prime}+\tau_{t} M_{t}$ dollars into the next day, where $m_{t}^{\prime}$ is money left over after trading and $\tau_{t} M_{t}$ is the lump sum transfer. We impose $x \geq 0$ and $m^{\prime} \geq 0$, but we do not impose $y \geq 0$. For technical reasons it is easier to allow $y<0$ when solving this problem, and then after finding an equilibrium, one can impose conditions to guarantee $y>0$; this is what we do here (see LW for a discussion).

The following result describes several useful features of the solution, including the linearity of $W(m)$.

Lemma 1 In the centralized market, for all agents and for all $t, x_{t}=x^{*}, m_{t}^{\prime}$ is independent of $m_{t}$, and $W_{m}=\phi_{t}$.

Proof: Substituting $y$ from (3) into (2), we have

$$
W(m)=\phi m+\max _{x, m^{\prime}}\left\{U(x)-x-\phi m^{\prime}+\beta V\left(m^{\prime}+\tau M\right)\right\}
$$

which implies that $W$ is linear in $m$ with slope $\phi$, and that the choices of $x$ and $m^{\prime}$ are independent of $m$. Differentiating, we get the first order conditions

$$
\begin{aligned}
1 & =U^{\prime}(x) \\
\phi & =\beta V_{m}\left(m^{\prime}+\tau M\right),
\end{aligned}
$$

the first of which implies $x=x^{*}$. 
The next step is to discuss the terms of trade in the decentralized market. In doublecoincidence meetings we adopt the symmetric Nash solution where the threat point of an agent with $m$ dollars is given by $W(m)$. It is straightforward to show that, for an agent with $m$ dollars, this implies $B(m, \tilde{m})=u\left(q^{*}\right)-c\left(q^{*}\right)+W(m)$; i.e., regardless of $(m, \tilde{m})$, in double-coincidence meetings agents produce the efficient quantity $q=q^{*}$ for each other and no money changes hands. In single-coincidence meetings we use the generalized Nash solution, with $\theta$ denoting the bargaining power of the buyer, and again the threat point of an agent with $m$ dollars given by $W(m)$. The solution is characterized in the next lemma, where we write $q=q(s, \tilde{s})$ and $d=d(s, \tilde{s})$ since then we can use the same notation in models where $s$ contains more than just $m$.

Lemma 2 In single coincidence meetings in the decentralized market, for all t, the bargaining solution is

$$
q_{t}(s, \tilde{s})=\left\{\begin{array}{ll}
q^{*} & \text { if } m \geq m_{t}^{*} \\
\tilde{q}_{t}(m) & \text { if } m<m_{t}^{*}
\end{array} \quad \text { and } d(s, \tilde{s})= \begin{cases}m_{t}^{*} & \text { if } m \geq m_{t}^{*} \\
m & \text { if } m<m_{t}^{*}\end{cases}\right.
$$

where $\tilde{q}_{t}(m)$ solves $g(q)=\phi_{t} M$, with

$$
g(q) \equiv \frac{\theta c(q) u^{\prime}(q)+(1-\theta) u(q) c^{\prime}(q)}{\theta u^{\prime}(q)+(1-\theta) c^{\prime}(q)}
$$

and $m_{t}^{*}=g\left(q^{*}\right) / \phi_{t}$.

Proof: This is a special case of the bargaining solution in Lemma 5 below.

Figure 1 shows the solution. An important observation is that, since the function $g(q)$ depends only on exogenous objects, $q_{t}(m)$ is a fixed function of the buyer's real balances, $z_{t}=\phi_{t} m$. As long as $z_{t} \geq z^{*}$, in real terms the buyer spends $z^{*}$ and gets $q^{*}$, where $z^{*}=\phi_{t} m_{t}^{*}=g\left(q^{*}\right)=\theta c\left(q^{*}\right)+(1-\theta) u\left(q^{*}\right)$ is constant. If $z_{t}<z^{*}$ the buyer spends all his cash and gets $q<q^{*}$. Since $q$ and $d$ depend on $s$ and $\tilde{s}$ only through the buyer's money 


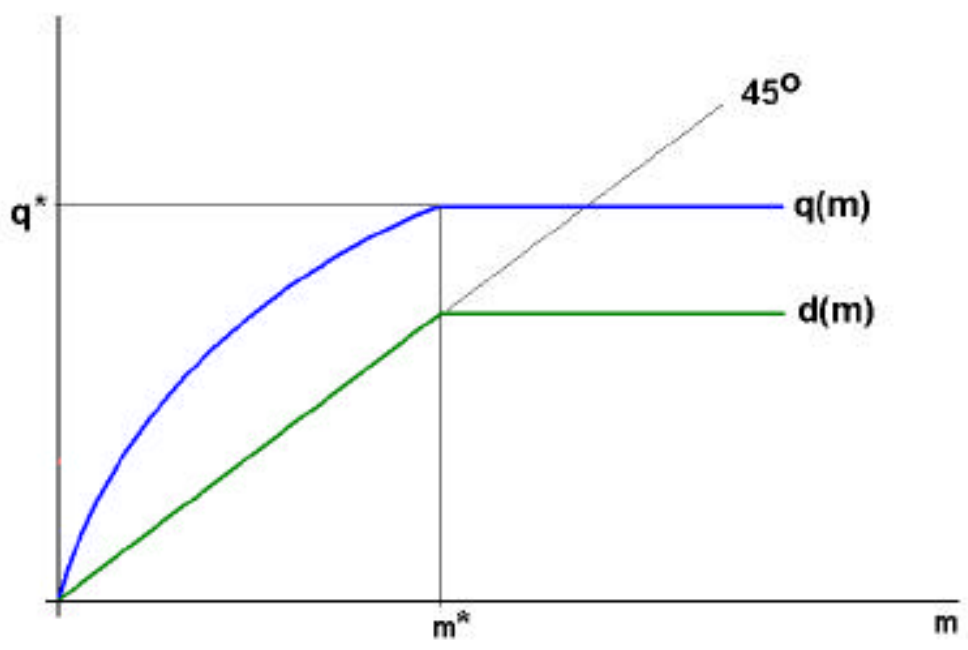

Figure 1: The Single-Coincidence Bargaining Solution

holdings, in what follows we write $q(s, \tilde{s})=q(m)$ and $d(s, \tilde{s})=d(m)$. Note that for $m<m^{*}$, $\tilde{q}$ is differentiable and $\tilde{q}^{\prime}(m)=\phi / g^{\prime}(q)$, where

$$
g^{\prime}=\frac{u^{\prime} c^{\prime}\left[\theta u^{\prime}+(1-\theta) c^{\prime}\right]+\theta(1-\theta)(u-c)\left(u^{\prime} c^{\prime \prime}-c^{\prime} u^{\prime \prime}\right)}{\left[\theta u^{\prime}+(1-\theta) c^{\prime}\right]^{2}}>0 .
$$

Using Lemmas 1 and 2 we can simplify (1) to

$$
V(s)=\alpha \sigma\{u[q(m)]-\phi d(m)\}+\alpha V_{0}+W(s),
$$

where again we write $s$ for $m$ so that we can use the same notation below, and

$$
V_{0}=\sigma \int\{-c[q(\tilde{m})]+\phi d(\tilde{m})\} d F(\tilde{m})+\Delta\left[u\left(q^{*}\right)-c\left(q^{*}\right)\right]
$$

does not depend on $m$. Given $u$ and $c$ are $\mathbb{C}^{n}, V$ is $\mathbb{C}^{n-1}$ for all $m \neq m^{*}$. For $m<m^{*}$,

$$
V_{m}=\alpha \sigma u^{\prime} \tilde{q}^{\prime}-\alpha \sigma \phi+\phi>0,
$$

and for $m>m^{*}, V_{m}=\phi$. A simple calculation shows that the limit of $V_{m}$ as $m \rightarrow m^{*}$ from below is strictly less than $\phi$, and so $V$ has a kink at $m^{*}$. For $m<m^{*}$ we have

$$
V_{m m}=\alpha \sigma \tilde{q}^{2} u^{\prime \prime}+\alpha \sigma u^{\prime} \tilde{q}^{\prime \prime}
$$


which cannot be signed in general since it depends on $q^{\prime \prime}$ which depends on $u^{\prime \prime \prime}$. However, it can be shown that $V_{m m}<0$ for all $m<m^{*}$ under the assumption that either $\theta$ is close to 1 or $u^{\prime}$ is log-concave (see LW).

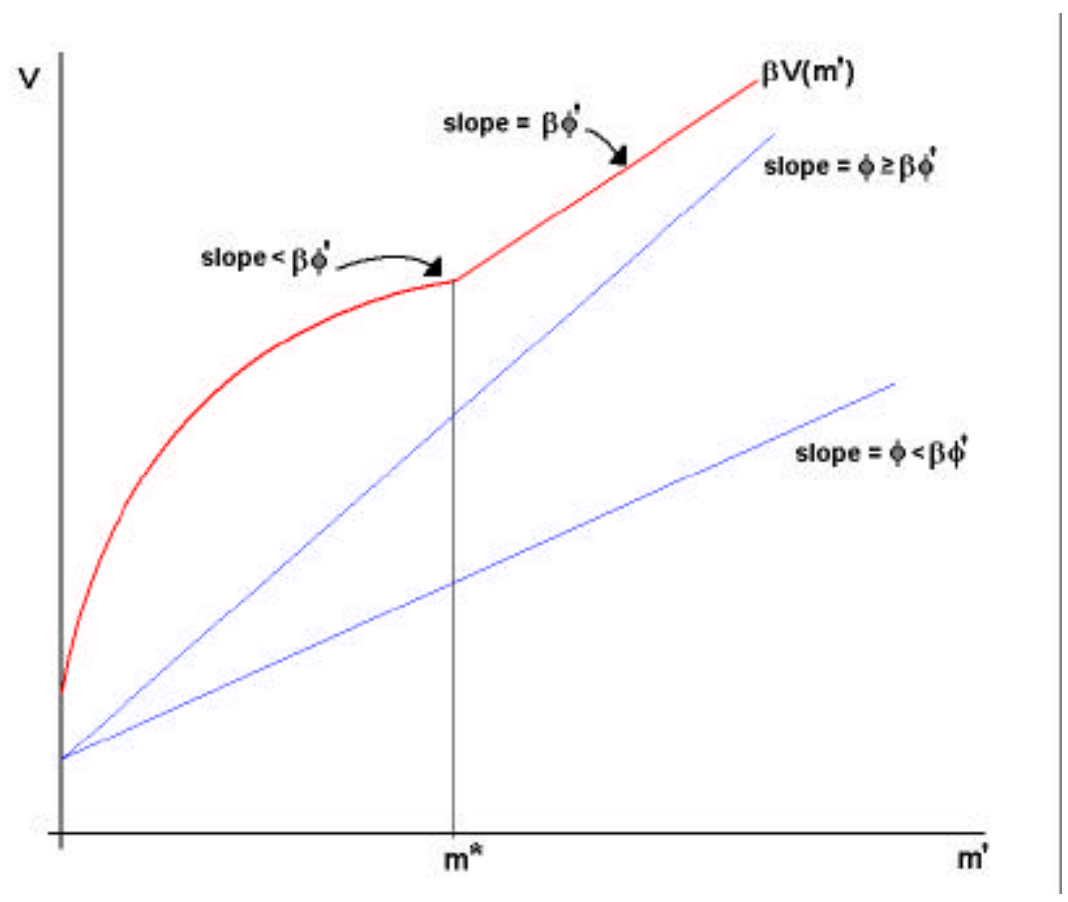

Figure 2: The Centralized Market Problem

Given all this, $V$ must be as shown in Figure 2, which illustrates the problem of deciding how much cash to take out of the centralized market at $t, \max \left\{-\phi_{t} m_{t+1}+\beta V_{t+1}\left(m_{t+1}\right)\right\}$. As should be clear from the picture, if $\phi_{t}<\beta \phi_{t+1}$ this problem has no solution. ${ }^{6}$ Hence, we can assume $\phi_{t} \geq \beta \phi_{t+1}$ without loss of generality. Then it is clear that any solution is to the left of $m_{t+1}^{*}$, simply because of the kink. This result does not depend at all on concavity, but under the assumptions stated above that guarantee $V_{m m}<0$ we have the additional result

\footnotetext{
${ }^{6}$ Formally, suppose $\phi_{t}<\beta \phi_{t+1}$ for some $t$, and consider the following arbitrage argument. At $t$, you can raise your general good production by $d y>0$ and sell it for $d y / \phi_{t}$ dollars. Then at $t+1$, you can use the money to reduce general good production by $d y \phi_{t+1} / \phi_{t}$ without changing anything else. The net utility gain from this is $d y\left(-1+\beta \phi_{t+1} / \phi_{t}\right)>0$; hence we cannot have $\phi_{t}<\beta \phi_{t+1}$. In this argument we did not worry about the possibility of $y_{t+1}=0$; however, it is not hard to show that $x_{t}>0$ for all $t$, and therefore $y_{t+1}>0$ for at least one agent in any equilibrium, and this is all that is necessary for the desired result.
} 
that there is a unique $m_{t+1}<m_{t+1}^{*}$ solving the problem. We summarize as follows.

Lemma 3 In the centralized market, for all $t$, all agents choose the same $m_{t+1}=m_{t}^{\prime}+\tau_{t} M_{t}$, and $m_{t+1}<m_{t+1}^{*}$.

Proof: Obvious from the discussion in the text.

This result is what makes the model simple. First, the result that all agents choose the same $m_{t+1}$ implies the distribution of money at the start of each day is degenerate at $m=M$. Second, the result that $m_{t+1}<m_{t+1}^{*}$ implies buyers spend all their money, so we know $d=M$ and $q=\tilde{q}(M)<q^{*}$. Hence, at the close of each decentralized market, the fraction $\alpha \sigma$ of agents who were buyers have 0 dollars, the fraction $\alpha \sigma$ who were sellers have $2 M$ dollars, and the rest have $M$ dollars. Since they all exit the centralized market holding $M$ and they all consume $x=x^{*}$, the budget constraint implies individual supply of $y$ is

$$
y= \begin{cases}x^{*}+\phi M & \text { for buyers } \\ x^{*}-\phi M & \text { for sellers } \\ x^{*} & \text { for others }\end{cases}
$$

where when we write "for buyers" we mean "for agents who were buyers in the previous subperiod" and so on. Aggregate supply is simply $y=x^{*}$.

At this stage we can consider the issue of nonnegativity. Recall that we have not imposed $y \geq 0$ so far. Given this we have shown that in equilibrium $x_{t}=x^{*}, m_{t+1}=M$ and $y$ is given by (??). We can guarantee $y \geq 0$ for all agents if we can be sure $x^{*} \geq \phi M=g(q)$, where $g$ is given in (7). Since $q<q^{*}$ and $g$ is monotonically increasing, we can guarantee what we want if we impose

$$
x^{*} \geq g\left(q^{*}\right)=\theta c\left(q^{*}\right)+(1-\theta) u\left(q^{*}\right)
$$

Hence, we have a simple condition to rule out $y<0$ in equilibrium. ${ }^{7}$

\footnotetext{
${ }^{7}$ This is true if we start by giving all agents the same endowment of money, $m_{0}$, or at least as long as the initial distribution $F_{0}$ is not too disperse. If some agent starts with a very large initial $m_{0}$ and we impose
} 
We now simplify things by reducing the model to one equation in one unknown. First, insert $V_{m}$ from (11) into (5), being careful to index all objects by the appropriate date, to get the following expression:

$$
\phi_{t}=\beta\left[\alpha \sigma u^{\prime}\left(q_{t+1}\right) \tilde{q}^{\prime}\left(m_{t+1}\right)+(1-\alpha \sigma) \phi_{t+1}\right] .
$$

Now substitute $\tilde{q}^{\prime}(q)=\phi / g^{\prime}(q)$ and $\phi=g(q) / m$ from the bargaining solution, as well as $m=M$, to arrive at

$$
\frac{g\left(q_{t}\right)}{M_{t}}=\beta \frac{g\left(q_{t+1}\right)}{M_{t+1}}\left[\alpha \sigma \frac{u^{\prime}\left(q_{t+1}\right)}{g^{\prime}\left(q_{t+1}\right)}+1-\alpha \sigma\right] .
$$

Given any exogenous path for $M_{t}$, this is a difference equation in $q_{t}$. An equilibrium can now be defined as a solution to (16) that stays in $\left[0, q^{*}\right]$ for all $t$; it is a monetary equilibrium if $q_{t}>0$ for all $t$.

Given the $q$ path, one can recover $\phi=g(q) / M$ and all of the other variables. The aggregate values of the centralized market variables are easy, since $y=x=x^{*}$, but we can also disaggregate into the amount $y$ supplied by buyers, sellers, and others as described in (13). We can compute the nominal price of a special good, $p^{s}=M / q$, and the general good, $p^{g}=1 / \phi$. It is immediate from (16) that the model displays classical neutrality: for any $\lambda>0$, if we change the money supply sequence so that $M_{t}$ becomes $\lambda M_{t}$ for all $t$, then all nominal variables $\left(\phi_{t}, p_{t}^{s}, p_{t}^{g}, \ldots\right)$ also change by a factor $\lambda$ while all real variables $\left(q_{t}, y_{t}, z_{t}, \ldots\right)$ stay the same. ${ }^{8}$ The model does not, however, display superneutrality: generally, changing

$y \geq 0$, he will set $y_{0}=0, x_{0}>x^{*}$ and $m_{1}>M$. Thus, his money holdings can stay above $M$ for several periods, but eventually any such agent will spent down his initial riches and then (??) guarantees $y \geq 0$ for all agents. To avoid this nuisance we can simply asume $F_{0}$ is not too disperse; to be precise, $y_{0} \geq 0$ if $x^{*} \geq g\left(q^{*}\right) m_{0} / M$, or

$$
m_{0} \leq \frac{M X^{*}}{\theta c\left(q^{*}\right)+(1-\theta) u\left(q^{*}\right)}
$$

for all agents. This condition on $F_{0}$ togther with (??) is sufficient to make nonnegativity a nonissue.

${ }^{8}$ More precisely, given a path for $M_{t}^{1}$, suppose there is a set of equilibrium paths for $q_{t}$, and for each $q_{t}$ there are associated values for each of the other real and nominal variables. Then for any $\lambda>0$, given the path $M_{t}^{2}=\lambda M_{t}^{1}$ the set of equilibrium paths for $q_{t}$ is the same, and for each $q_{t}$ the associated values of each the other real variables is the same while the associated values of each of the nominal variables changes by the factor $\lambda$. 
the growth rate of $M$ will affect at least some of the real variables, as we will discuss in detail below.

A case that makes sense when $\tau_{t}=\tau$ is constant is a steady state monetary equilibrium, which is a constant solution $q>0$ to $(16)$ with $M_{t+1}=(1+\tau) M_{t}$ :

$$
1=\frac{\beta}{1+\tau}\left[\alpha \sigma \frac{u^{\prime}(q)}{g^{\prime}(q)}+1-\alpha \sigma\right] .
$$

In a such an equilibrium $\phi_{t}=g(q) / M_{t}$ falls as $M_{t}$ grows, but real balances $z_{t}=\phi_{t} M_{t}=g(q)$ remain constant; i.e., the inflation rate equals the rate of monetary expansion, $\tau$. It is straightforward to establish the existence of a monetary steady state, and either uniqueness or multiplicity, depending on assumptions (see LW). In some special cases the analysis is especially easy; e.g., if $\theta=1$ (take-it-or-leave-it offers by the buyer) then (7) implies $g(q)=$ $c(q)$, and (17) is really quite simple.

In steady state, the general result $\beta \phi_{t+1} \leq \phi_{t}$ implies $\beta \phi_{t+1} M_{t+1} \leq \phi_{t} M_{t}(1+\tau)$, and hence $\beta \leq 1+\tau$. This puts a constraint on policy: we cannot contract the money supply faster than the so-called Friedman Rule, $\tau=\beta-1$; if we try, the monetary equilibrium will break down. It is not hard to check that $q$ is increasing in $\frac{\beta}{1+\tau}$, and that $q \rightarrow q^{*}$ as $\frac{\beta}{1+\tau} \rightarrow 1$ iff $\theta=1$. This implies that the Friedman Rule is the optimal policy, since it gets $q$ as close to $q^{*}$ as possible before the equilibrium breaks down, but it cannot achieve the fully efficient outcome $q=q^{*}$ unless $\theta=1$. This can have interesting implications for some issues, including the welfare cost of inflation (see LW for further discussion).

Although the equilibrium is inefficient if we have either $\tau>\beta-1$ or $\theta<1$, this inefficiency manifests itself only in the decentralized market - in the centralized market agents always consume the efficient quantity $x^{*}$. Indeed, the model displays a very strong dichotomy: one can solve independently for the allocations in the decentralized and centralized markets. That is, at least the aggregate allocation in the centralized market, $x=y=x^{*}$, is independent of the solution $q$ to (16), and vice-versa. The value of money $\phi=g(q) / M$ does depend on $q$, 
and this does affect how much $y$ different individuals supply as seen in (13), but this does not affect aggregate supply. Hence, for example, an increase in $\tau$ will lower $q$ but does has no effect on $x$ or $y$.

This completes our review of the basic LW model. We close the section by sketching an alternative version where, instead of assuming that $C(y)=y$ is linear, we assume that $C^{\prime \prime}(y)>0$ and that $U(x)=x$ is linear. With this specification the centralized market problem becomes

$$
W(m)=\max _{x, y, m^{\prime}} x-C(y)+\beta V\left(m^{\prime}+\tau M\right)
$$

subject to (3). We do not impose $x \geq 0$ here, for the same reason we did not impose $y \geq 0$ earlier. Substituting for $x$ from (3) and differentiating with respect to $y$ and $m^{\prime}$, we get

$$
\begin{aligned}
1 & =C^{\prime}(y) \\
\phi & =\beta V_{m}\left(m^{\prime}+\tau M\right) .
\end{aligned}
$$

Hence, $y=y^{*}$ where $C^{\prime}\left(y^{*}\right)=1$, and $m^{\prime}$ satisfies the same condition as before.

In the original model $x=x^{*}$ is constant across individuals and $y$ varies according to whether an agent was a buyer or seller in the previous subperiod, while here $y=y^{*}$ is constant and $x$ varies according to:

$$
x= \begin{cases}y^{*}-\phi M & \text { for buyers } \\ y^{*}+\phi M & \text { for sellers } \\ y^{*} & \text { for others }\end{cases}
$$

We can guarantee $x \geq 0$ with a condition like (??), except $y^{*}$ replaces $x^{*}$. Otherwise, things are exactly the same. In any case, we summarize the key result for our purposes as follows:

Proposition 1 The basic model dichotomizes: one can solve for the equilibrium path of $q$ and the equilibrium path of aggregate $(x, y)$ independently, and monetary policy affects the former but not the latter. 


\section{Capital}

Here we introduce capital and neoclassical production. As in the standard one-sector growth model, capital is the same as the general consumption good. Later we introduce firms explicitly, but for now we let each agent have access to a technology for producing general goods $f(k)$, with the usual properties $f(0)=0, f^{\prime}>0$ and $f^{\prime \prime}<0$. A very important assumption is that one's capital is not mobile: it can be traded in the centralized market, but cannot be carried into the decentralized market. Nor can claims to capital be traded in the decentralized market, since agents are anonymous and hence could renege on any such claim without fear of retribution. These assumptions are made simply to guarantee that capital or claims to capital do not replace money as a medium of exchange - that is, to guarantee that money is still essential. ${ }^{9}$

The individual state variable now includes one's money holdings and capital stock, $s=$ $(m, k)$, with joint distribution $F(s)$. In this environment, Bellman's equation is the natural generalization of (1):

$$
\begin{aligned}
V(s)= & \alpha \sigma \int\{u[q(s, \tilde{s})]+W[m-d(s, \tilde{s}), k]\} d F(\tilde{s}) \\
& +\alpha \sigma \int\{-c[q(\tilde{s}, s)]+W[m+d(\tilde{s}, s), k]\} d F(\tilde{s}) \\
& +\alpha \Delta \int B(s, \tilde{s}) d F(\tilde{s})+(1-2 \alpha \sigma-\alpha \Delta) W(s) .
\end{aligned}
$$

Again, the aggregate state $F$ and the date $t$ are implicit in the notation, but we emphasize again that we are not imposing stationarity. The centralized market problem is

$$
\begin{aligned}
& W(s)=\max _{x, m^{\prime}, k^{\prime}} x+\beta V\left(m^{\prime}+\tau M, k^{\prime}\right) \\
& \text { s.t. } x=\phi\left(m-m^{\prime}\right)+f(k)+(1-\delta) k-k^{\prime},
\end{aligned}
$$

\footnotetext{
${ }^{9}$ Obviously modeling at a deeper level the restriction that capital cannot be traded in the decenteralized market may be worthwhile, and presumably it would be interesting to have some capital or claims to capital circulate along side of currency. One possible route is to assume some agents are anonymous in the decentralized market while others are not, along the lines of Cavalcante and Wallace (1999), perhaps.
} 
where $\delta$ is the depreciation rate. Notice we are using the version of the model in the previous section with linear $U(x)=x$, and as was the case there we do not impose $x \geq 0$, but we can check that this is true once we find an equilibrium.

We have the following versions of Lemmas 1 and 2 .

Lemma 4 In the centralized market with capital, for all agents and for all $t, m^{\prime}$ and $k^{\prime}$ are independent of $s=(m, k), W_{m}=\phi$ and $W_{k}=f^{\prime}+1-\delta$.

Proof: Substituting from (24) into (23), we have

$$
W(s)=\phi m+f(k)+(1-\delta) k+\max _{m^{\prime}, k^{\prime}}\left\{-\phi m^{\prime}-k^{\prime}+\beta V\left(m^{\prime}+\tau M, k^{\prime}\right)\right\}
$$

and everything follows. In particular,

$$
\begin{aligned}
1 & =\beta V_{k}\left(m^{\prime}+\tau M, k^{\prime}\right) \\
\phi & =\beta V_{m}\left(m^{\prime}+\tau M, k^{\prime}\right) .
\end{aligned}
$$

are the first order conditions.

Lemma 5 In the model with capital, for all t, the single-coincidence bargaining solution is exactly the same as in Lemma 2.

Proof: The generalized Nash problem when the buyer has $s=(m, k)$ and the seller $\tilde{s}=(\tilde{m}, \tilde{k})$ is

$$
\max _{q, d}[u(q)+W(m-d, k)-W(m, k)]^{\theta}[-c(q)+W(\tilde{m}+d, \tilde{k})-W(\tilde{m}, \tilde{k})]^{1-\theta}
$$

subject to $d \leq m$. Lemma 4 implies $W(m-d, k)-W(m, k)=-\phi d$ and $W(\tilde{m}+d, \tilde{k})-$ $W(\tilde{m}, \tilde{k})=\phi d$, and so this problem reduces to

$$
\max _{q, d}[u(q)-\phi d]^{\theta}[-c(q)+\phi d]^{1-\theta}
$$


subject to $d \leq m$. Notice $\tilde{m}, k$ and $\tilde{k}$ have vanished.

The Kuhn-Tucker conditions, which are necessary and sufficient here, are

$$
\begin{aligned}
\frac{\theta u^{\prime}(q)}{u(q)-\phi d}-\frac{(1-\theta) c^{\prime}(q)}{-c(q)+\phi d} & =0 \\
\frac{-\theta \phi}{u(q)-\phi d}+\frac{(1-\theta) \phi}{-c(q)+\phi d}-\lambda & =0 \\
\lambda(m-d) & =0
\end{aligned}
$$

where $\lambda \geq 0$ is the multiplier. If $\lambda=0$, the first two conditions yield $u^{\prime}(q)=c^{\prime}(q)$, or $q=q^{*}$, and then $d=m^{*}$. If $\lambda>0$, the solution is $d=m$ and $q=\tilde{q}(m)$, where $\tilde{q}(m)$ solves the first Kuhn-Tucker condition, which can be rearranged into $m \phi=g(q)$ with $g$ defined in (7). By the implicit function theorem, $\tilde{q}^{\prime}>0$. Hence, for $m<m^{*}$ we have $d=m$ and $q=\tilde{q}(m)$ and for $m \geq m^{*}$ we have $d=m^{*}$ and $q=q^{*}$.

Since the bargaining solution here depends on $m$ but not $\tilde{m}, k$ or $\tilde{k}$, we again write $q=q(m)$ and $d=d(m)$. Hence, Bellman's equation again reduces to $(9)$ in the previous section and $V_{m}$ and $V_{m m}$ are again given by (11) and (12). The conditions that guarantee $V$ is strictly concave in $m$ from the previous section ( $\theta \approx 1$ or $u^{\prime}$ log-concave) still apply, and this yields the generalized version of Lemma 3.

Lemma 6 In the centralized market with capital, for all $t$, all agents choose the same $m_{t}^{\prime}$ and $k_{t}^{\prime}$, and $m_{t+1}=m_{t}^{\prime}+\tau_{t} M_{t}<m_{t+1}^{*}$.

Proof: Given $V_{m m}<0, V_{m k}=0$ and $V_{k k}=f^{\prime \prime}<0$ imply that $V$ is strictly concave, and hence there exists a unique solution to (25) and (26). The result $m_{t+1}<m_{t+1}^{*}$ follows from the same argument used in Lemma 3.

As in the previous section, $F$ is degenerate at $(m, k)=(M, K)$, although here of course the aggregate capital stock $K$ is endogenous, and buyers spend all their money in every 
single-coincidence meeting, $d=M$. In equilibrium we have the following version of $(21)$ :

$$
x= \begin{cases}X-\phi M & \text { for buyers } \\ X+\phi M & \text { for sellers } \\ X & \text { for others }\end{cases}
$$

where $X=f(K)+(1-\delta) K-K^{\prime}$. Thus, individual consumption in the centralized market depends on whether one spent or acquired money in the previous subperiod, but aggregate consumption is simply $X$.

In the previous section we reduced the model to one equation by substituting $V_{m}$ into the first order condition for $m^{\prime}$ and then inserting the bargaining solution. The same procedure here yields exactly the same result, which we repeat for convenience:

$$
\frac{g\left(q_{t}\right)}{M_{t}}=\beta \frac{g\left(q_{t+1}\right)}{M_{t+1}}\left[\alpha \sigma \frac{u^{\prime}\left(q_{t+1}\right)}{g^{\prime}\left(q_{t+1}\right)}+1-\alpha \sigma\right] .
$$

Similarly, substituting $V_{k}$ into $(25)$ we get

$$
1=\beta\left[f^{\prime}\left(K_{t+1}\right)+1-\delta\right]
$$

This is the familiar condition from the standard (nonmonetary) neoclassical growth model. ${ }^{10}$ Equilibrium can now be defined as a path $(q, K)$ that solves $(28)$ and (29) subject to the usual side conditions, $K_{0}$ is given, $q \in\left[0, q^{*}\right]$, and $k \in[0, \bar{K}]$ where as is standard $\bar{K}$ is the maximum of $K_{0}$ and the upper bound on the sustainable capital stock.

The main point is that when we introduce capital the model still dichotomizes: (28) and (29) can be solved independently. The set of equilibrium $q$ paths is the same as in the basic LW model while the $K$ path is the same as in a nonmonetary growth model. As in the model from the previous section, $q$ affects $\phi$ and hence individual consumption in the centralized

\footnotetext{
${ }^{10}$ That is, the familiar condition when utility is linear, $U(x)=x$. Normally, $U^{\prime}\left(x_{t}\right)$ appears on the left and $U^{\prime}\left(x_{t+1}\right)$ on the right side of the Euler equation; these cancel in this case not only in steady state but for all $t$. This implies we jump to steady state in one period, ignoring nonnegativity constraints on $x$, which is valid if $f\left(K_{0}\right)+(1-\delta) K_{0} \geq K^{s}$ where $K^{s}$ is the steady state. We emphasize that this has nothing to do with money and also holds in the standard growth model with $U(x)=x$. In the next section $U$ will be strictly concave and hence we do not jump to the steady state immediately.
} 
market as seen in (27), but aggregate consumption $X=f(K)+(1-\delta) K-K^{\prime}$ is independent of $\phi$ and $q$. In terms of policy implications, for example, in this model inflation will affect the value of money in decentralized trade, but not aggregate consumption or investment in the general goods market.

We summarize as follows.

Proposition 2 The model with capital dichotomizes: one can solve for the equilibrium path of $q$ and the equilibrium path of aggregate $(X, K)$ independently; monetary policy affects the former but not the latter.

\section{Capital and Labor}

The previous section may help move search-based monetary theory somewhat towards the mainstream, but does not go all the way. In this section, instead of having agents produce general goods themselves, we assume there is a representative firm with a constant returns to scale production function $f(K, H, Z)$ that hires capital $K$ at rate $r$ and labor $H$ at wage $w$. The state of technology $Z$ evolves exogenously according to $Z_{t+1}=\zeta\left(Z_{t}, \varepsilon_{t}\right)$, where $\varepsilon$ is an i.i.d. random technology shock observed at the start of period $t$. Every night, individuals supply labor and capital and buy general goods in the centralized market. We assume utility is separable and linear in leisure, given by $1-h$ (total time is 1 and $h$ is hours worked). If there were no decentralized trade or money, this would be a standard macroeconomic model - indeed, except for some minor differences in notation it would be identical to the model in Hansen (1985). ${ }^{11}$

As Section 3, during the day agents meet in a decentralized market where they cannot bring their capital, nor can they trade claims to capital because of anonymity. Bellman's

\footnotetext{
${ }^{11}$ Hansen (1985) does not directly assume linearity, but uses Rogerson's (1988) indivisible labor model with lotteries to derive a reduced-form utility function that is linear in hours worked. In terms of its macro implications this is of no consequence, and one can view the linearity in that model as a primitive. Alternatively one could also reinterpret the linearity in our model in terms indivisible labor and lotteries.
} 
equation is again given by $(22)$, except now the aggregate state is $(Z, F)$ but in any case this is subsumed in the notation. The centralized market problem is

$$
\begin{aligned}
& W(s)=\max _{x, h, m^{\prime}, k^{\prime}} U(x)+A(1-h)+\beta E V\left(m^{\prime}+\tau M, k^{\prime}\right) \\
& \text { s.t. } x=\phi\left(m-m^{\prime}\right)+w h+r k+(1-\delta) k-k^{\prime},
\end{aligned}
$$

where the expectation is with respect to future prices. As is standard, profit maximization implies these prices will satisfy $w=f_{h}(K, H, Z)$ and $r=f_{k}(K, H, Z)$ in equilibrium. Note that we do not impose $h \geq 0$ here, for the same reason we did not impose $y \geq 0$ or $x \geq 0$ in the earlier models, but we can check that this is true later.

Lemma 7 In the centralized market with capital and labor, for all agents and for all $t, x$, $k^{\prime}$ and $m^{\prime}$ are independent of $s=(m, k), W_{m}=\frac{A}{w} \phi$ and $W_{k}=\frac{A}{w}(r+1-\delta)$.

Proof: Substituting for $h$ from (31) into (30), we have

$$
\begin{aligned}
W(s)= & A+\frac{A}{w}[\phi m+(r+1-\delta) k] \\
& +\max _{x, m^{\prime}, k^{\prime}}\left\{U(x)-\frac{A}{w}\left(x+\phi m^{\prime}+k^{\prime}\right)+\beta E V\left(m^{\prime}+\tau M, k^{\prime}\right)\right\},
\end{aligned}
$$

and everything follows. In particular,

$$
\begin{aligned}
U^{\prime}(x) & =A / w \\
A / w & =\beta E V_{k}\left(m^{\prime}+\tau M, k^{\prime}\right) \\
\phi A / w & =\beta E V_{m}\left(m^{\prime}+\tau M, k^{\prime}\right) .
\end{aligned}
$$

are the first order conditions.

Lemma 8 In the model with capital and labor, for all t, the single-coincidence bargaining solution is the same as in Lemma 2 except now $\tilde{q}_{t}(m)=g^{-1}\left(\frac{A \phi_{t}}{w_{t}} m\right)$ where $g$ is still given by (7) and $\frac{A}{w} \phi m_{t}^{*}=g\left(q^{*}\right)$. 
Proof: Lemma 7 reduces the generalized Nash problem to

$$
\max _{q, d}\left[u(q)-\frac{A \phi}{w} d\right]^{\theta}\left[-c(q)+\frac{A \phi}{w} d\right]^{1-\theta}
$$

subject to $d \leq m$. A argument involving Kuhn-Tucker conditions similar to the one in Lemma 5 completes the proof.

Earlier, $m^{*}$ and $\tilde{q}$ depended only on $z=\phi m$, or real balances denominated in the general good; now they depend on real balances denominated in leisure units, $\frac{A}{w} \phi m$, and we have $\tilde{q}^{\prime}=\frac{A}{w} \phi / g^{\prime}$. Otherwise, the bargaining solution is the same as before. Bellman's equation becomes

$$
V(s)=\alpha \sigma\left\{u[q(m)]-\frac{A}{w} \phi d(m)\right\}+\alpha V_{0}+W(s)
$$

where $V_{0}$ looks like $(10)$ in the previous section except that $\frac{A}{w} \phi$ replaces $\phi$. Hence, $V_{m}$ and $V_{m m}$ are given by expressions that look like (11) and (12) except that $\frac{A}{w} \phi$ replaces $\phi$. The conditions that guarantee $V$ is strictly concave still apply, and so we have:

Lemma 9 In the centralized market with capital and labor, for all $t$, all agents choose the same $m_{t}^{\prime}$ and $k_{t}^{\prime}$, and $m_{t+1}=m_{t}^{\prime}+\tau M_{t}<m_{t+1}^{*}$.

\section{Proof: Similar to Lemma 3.}

We again have a degenerate distribution $F$, and agents spend all their money in singlecoincidence meetings. From (32) all agents consume $x_{t}=X_{t}=U^{\prime-1}\left(\frac{A}{w_{t}}\right)$, which depends on $t$ through the wage but does not depend on the individual state. What differs across agents in this model is individual labor supply, given by

$$
h= \begin{cases}H+\frac{\phi}{w} M & \text { for buyers } \\ H-\frac{\phi}{w} M & \text { for sellers } \\ H & \text { for others }\end{cases}
$$

where $H$ is aggregate labor supply,

$$
H=\frac{1}{w}\left[x^{*}+k^{\prime}-(r+1-\delta) k\right] .
$$


Hence, $H_{t}$ may depend on $t$, but given $t$ all individuals supply $H_{t}$ plus an adjustment to bring their money holdings to $m=M$.

To simplify this version of the model, first insert $V_{m}$ into (34)

$$
\phi A / w=\beta E\left[\alpha \sigma u^{\prime}(q) \tilde{q}^{\prime}(m)+(1-\alpha \sigma) \frac{A}{w} \phi\right]
$$

Then insert $\phi A / w=g(q) / m$ and $\tilde{q}^{\prime}(m)=g(q) / m g^{\prime}(q)$ to derive

$$
\frac{g\left(q_{t}\right)}{M_{t}}=\beta E\left[\alpha \sigma \frac{u^{\prime}\left(q_{t+1}\right)}{g^{\prime}\left(q_{t+1}\right)} \frac{g\left(q_{t+1}\right)}{M_{t+1}}+(1-\alpha \sigma) \frac{g\left(q_{t+1}\right)}{M_{t+1}}\right]
$$

We can drop the expectation operator, since nothing on the right hand side is random. Hence we are right back to (16), which we again repeat for convenience:

$$
\frac{g\left(q_{t}\right)}{M_{t}}=\beta \frac{g\left(q_{t+1}\right)}{M_{t+1}}\left[\alpha \sigma \frac{u^{\prime}\left(q_{t+1}\right)}{g^{\prime}\left(q_{t+1}\right)}+1-\alpha \sigma\right] .
$$

Once again, we can solve for the path of $q$, independently of the other endogenous variables in the model.

To find the conditions the other variables must satisfy, insert $V_{k}=W_{k}=\frac{A}{w}(r+1-\delta)$ and the equilibrium conditions $w=f_{h}$ and $r=f_{k}$ into the remaining first order conditions (32) and (33) to yield

$$
\begin{aligned}
A & =U^{\prime}\left(X_{t}\right) f_{h}\left(K_{t}, H_{t}, Z_{t}\right) \\
U^{\prime}\left(X_{t}\right) & =\beta E U^{\prime}\left(X_{t+1}\right)\left[f_{k}\left(K_{t+1}, H_{t+1}, Z_{t+1}\right)+1-\delta\right]
\end{aligned}
$$

where $X_{t}=H_{t} f_{h}+K_{t} f_{k}+(1-\delta) K_{t}-K_{t+1}$ is aggregate consumption. As is standard, by Euler's Theorem

$$
X_{t}=f\left(K_{t}, H_{t}, Z_{t}\right)+(1-\delta) K_{t}-K_{t+1} .
$$

Of course, (39), (40) and (41) are nothing more nor less than the standard equations characterizing equilibrium paths for $\left(X_{t}, H_{t}, K_{t+1}\right)$ in the stochastic growth model without money. 
An equilibrium can be defined here in the obvious way (paths for the endogenous variables satisfying the conditions derived above, plus the usual side conditions, such as a given value for the initial capital stock $K_{0}$ ). The main point is that the model still dichotomizes: the set of equilibrium $q$ paths is the same as in the basic LW model and the other real variables are the same as in the nonmonetary growth model. As before, $q$ affects $\phi$ and hence, in this version, individual labor supply, but not aggregate labor supply, and not consumption or investment, in the centralized market.

We summarize as follows:

Proposition 3 The model with capital and labor dichotomizes: one can solve for the equilibrium path of $q$ and the equilibrium path of aggregate $(X, H, K)$ independently; monetary policy affects the former but not the latter.

\section{Shopping Time}

In this section, we allow agents to choose their search intensity, or equivalently, their shopping time in the decentralized market. One reason is that one can find in the literature models where it is simply assumed that individuals have to spend time shopping, where the required amount of time to purchase a given consumption bundle is some arbitrary decreasing function of real balances (see Walsh [1998]). Another reason is to see what it does to the dichotomy results. Thus, we assume an increase in time spent shopping, or in search effort, $l$, increases one's arrival rate $\alpha=\alpha(l)$ in the decentralized market but reduces the time left available for leisure or labor. ${ }^{12}$ We assume $\alpha^{\prime}>0$ and $\alpha^{\prime \prime}<0$. Here leisure is $1-l-h$. Otherwise things are the same as the previous section.

The state variable when an agent enters the decentralized market is again $s=(m, k)$,

\footnotetext{
${ }^{12}$ It is not hard to derive the relationship $\alpha(l)$ from an underlying matching technology that takes the search intensity of agents as inputs, and every agent chooses $l$ taking as given the aggregate search intensity $L$; see Berensten, Rocheteau and Shi (2001), e.g., for details.
} 
and Bellman's equation is now given by

$$
\begin{aligned}
V(s)= & \alpha(l) \sigma \int\{u[q(s, \tilde{s})]+W[m-d(s, \tilde{s}), k, l]\} d F(\tilde{s}) \\
& +\alpha(l) \sigma \int\{-c[q(\tilde{s}, s)]+W[m+d(\tilde{s}, s), k, l]\} d F(\tilde{s}) \\
& +\alpha(l) \Delta \int B(\tilde{s}, s) d F(\tilde{s})+[1-2 \alpha(l) \sigma-\alpha(l) \Delta] W(m, k, l)
\end{aligned}
$$

which is identical to (22) except for the fact that the arrival rate $\alpha$ is a function of $l$, and $l$ is an argument of $W$ since total time left to allocate between leisure and labor in the centralized market is $1-l$. The problem in the centralized market is

$$
W(m, k, l)=\max _{x, m^{\prime}, k^{\prime}, h} U(x)+A(1-l-h)+\beta E V\left(m^{\prime}+\tau M, k^{\prime}\right)
$$

subject to (31). Again we do not impose $h \geq 0$.

The generalized versions of Lemmas 7 and 8 are:

Lemma 10 In the centralized market with capital, labor and shopping, for all agents and for all $t, x, k^{\prime}$ and $m^{\prime}$ are independent of $s=(m, k), W_{m}=\underset{w}{A} \phi, W_{k}=\underset{w}{A}(r+1-\delta)$, and $W_{l}=-A$.

Proof: Substituting for $h$ from (31) into (43), we have

$$
\begin{aligned}
W(m, k, l)= & A(1-l)+\frac{A}{w}[\phi m+(r+1-\delta) k] \\
& +\max _{x, m^{\prime}, k^{\prime}}\left\{U(x)-\frac{A}{w}\left(x+\phi m^{\prime}+k\right)+\beta E V\left(m^{\prime}+\tau M, k^{\prime}\right)\right\},
\end{aligned}
$$

and everything follows. The first order conditions are the same as in the previous section, (32), (33) and (34).

Lemma 11 In the model with capital, labor and shopping, for all t, the single-coincidence bargaining solution is the same as in Lemma 8. 
Proof: Obvious.

The usual procedure implies we can write Bellman's equation as

$$
V(m, k)=\alpha(l) \sigma\left\{u[q(m)]-\frac{A}{w} \phi d(m)\right\}+\alpha(l) V_{0}+W(m, k, l)
$$

where $V_{0}$ is the same as above. The only difference from the previous section is that $l$ is endogenous, and as such it must satisfy the first order condition

$$
\alpha^{\prime}(l) \sigma\left\{u[q(m)]-\frac{A}{w} \phi d(m)\right\}+\alpha^{\prime}(l) V_{0}+W_{l}=0
$$

Then we have:

Lemma 12 In the model with capital, labor and shopping, for all $t$, in the decentralized market all agents choose the same $l_{t}$, and in the centralized market all agents choose the same $m_{t}^{\prime}$ and $k_{t}^{\prime}$, and $m_{t+1}=m_{t}^{\prime}+\tau M_{t}<m_{t+1}^{*}$.

Proof: Given $\alpha^{\prime \prime}<0$ and $W_{l}=-A$, there is a unique $l$ solving (44). The rest is similar to the proof of Lemma 3.

We again have a degenerate distribution $F$, and agents spend all their money in singlecoincidence meetings. Combining these results with (44), in equilibrium we have

$$
\alpha^{\prime}(l) \sigma\{u[q(M)]-c[q(M)]\}+\alpha^{\prime}(l) \Delta\left[u\left(q^{*}\right)-c\left(q^{*}\right)\right]=A
$$

Other than determining $l$ the model works the same as in the previous section. Individuals still supply more or less labor at night depending on what happened during the day, but all leave the centralized night market with the same $m=M$. Again, we did not impose $h \geq 0$, but conditions can be assumed to guarantee this is true in equilibrium.

While there the may be good reasons for endogenizing search effort, in general, it does nothing to change our neoclassical dichotomy. Here we can solve the system

$$
\begin{aligned}
\frac{g\left(q_{t}\right)}{M_{t}} & =\beta \frac{g\left(q_{t+1}\right)}{M_{t+1}}\left[\alpha\left(l_{t}\right) \sigma \frac{u^{\prime}\left(q_{t+1}\right)}{g^{\prime}\left(q_{t+1}\right)}+1-\alpha\left(l_{t}\right) \sigma\right] \\
A & =\alpha^{\prime}\left(l_{t}\right)\left\{\sigma\left[u\left(q_{t}\right)-c\left(q_{t}\right)\right]+\Delta\left[u\left(q^{*}\right)-c\left(q^{*}\right)\right]\right\}
\end{aligned}
$$


for the equilibrium paths of $(q, l)$ independently of the paths of $(X, K, H)$, which are still determined by the usual conditions for the nonmonetary growth model, (39), (40) and (41) in the previous section. One thing this does illustrate is that our neoclassical dichotomy in general does not say we can solve for $q$ with a single equation independent of the rest of the system; it says we can solve for variables determined in the decentralized market independent of variables in the centralized market.

Proposition 4 The model with capital, labor and shopping dichotomizes: one can solve for the equilibrium path of $(q, l)$ and the equilibrium path of $\left(X, H, K^{\prime}\right)$ independently; monetary policy affects the former but not the latter.

\section{Conclusion}

This paper pursues the integration of search-based monetary theory and standard macroeconomics. The setup in Lagos and Wright (2002a) was extended by introducing neoclassical production and having capital and labor trade in the centralized markets. The result is a tractable framework that nests the search model and a simple neoclassical growth model. A strong dichotomy emerges in the versions we considered: one can solve for the outcome in the decentralized markets and the outcome in the centralized markets independently. The value of money and hence monetary policy determines output and consumption in decentralized exchange, but this does not affect aggregate employment, investment, or consumption of general goods. We do not claim the dichotomy will hold in all possible versions of the model, but we emphasize that we did not "rig" things to get the result - indeed, it was a surprise. In future work, it may be interesting to investigate what features of similar models do or do not lead to this kind of dichotomy. 


\section{References}

[1] Azariadis, C. Intertemporal Macroeconomics. Oxford: Blackwell, 1993.

[2] Berentsen, Aleksander, Guillaume Rocheteau and Shouyong Shi. "Friedman Meets Hosios: Efficiency in Search Models of Money." Unpublished manuscript, 2001.

[3] Berentsen, Aleksander, Guillaume Rocheteau and Christopher Waller. "Money and Specialization." Unpublished manuscript, 2002.

[4] Berentsen, Aleksander, Ricardo Lagos and Guillaume Rocheteau. "Mechanism Design in Monetary Models." Unpublished manuscript, 2002.

[5] Cavalcanti, Ricardo and Neil Wallace. "Inside and Outside Money as Alternative Media of Exchange." Journal of Money, Credit, and Banking, August 1999, 31, 443-57.

[6] Diamond, Peter A. "Aggregate Demand Management in Search Equilibrium," Journal of Political Economy, 1982.

[7] Faig, Miguel (2001) "A Search Theory of Money and Comerce with Neoclassical Production." Unpublished manuscript, 2001.

[8] Hansen, Gary D. "Indivisible Labor and the Business Cycle." Journal of Monetary Economics, 1985.

[9] Kiyotaki, Nobuhiro and John Moore. "Liquidity, Business Cycles and Monetary Policy." Lecture 2, Clarendon Lectures, November 2001.

[10] Kiyotaki, Nobuhiro and Randall Wright. "On Money as a Medium of Exchange." Journal of Political Economy 97, 1989, 927-954.

[11] Kiyotaki, Nobuhiro and Randall Wright. "A Search-Theoretic Approach to Monetary Economics." American Economic Review 83, 1993, 63-77.

[12] Kocherlakota, Narayana. "Money is Memory." Journal of Economic Theory 81, 1998, 232-251.

[13] Lagos, Ricardo and Randall Wright "A Unifed Framework for Monetary Theory and Policy Evaluation." Unpublished manuscript, 2002a.

[14] Lagos, Ricardo and Randall Wright "Dynamics, Cycles and Sunspot Equilibria in 'Genuinely Dynamic, Fundamentally Disaggregative' Models of Money." Unpublished manuscript, 2002b. 
[15] Molico, Miguel. "The Distribution of Money and Prices in Search Equilibrium." Unpublished manuscript, 1999.

[16] Rocheteau, Guillaume and Randall Wright. "Money in Search Equilibrium, in Competitive Equilibrium, and in Competitive Search Equilibrium." Unpublished manuscript, 2002.

[17] Rogerson, Richard. "Indivisible Labor, Lotteries and Equilibrium," Journal of Monetary Economics 66, 1988.

[18] Sargent, Thomas J. Macroeconomic Theory. New York: Academic Press, 1979.

[19] Shi, Shouyong. "Money and Prices: A Model of Search and Bargaining." Journal of Economic Theory 67, 1995, 467-496.

[20] Shi, Shouyong. "A Divisible Search Model of Fiat Money." Econometrica 64, 1997, 75-102.

[21] Shi, Shouyong. "Search, Money, and Capital." Journal of Monetary Economics 66, 1999, 81-103.

[22] Trejos, Alberto and Randall Wright. "Search, Bargaining, Money and Prices." Journal of Political Economy 103, 1995, 118-141.

[23] Walsh, Carl E. Monetary Theory and Policy. Cambridge: MIT Press, 1998.

[24] Wallace, Neil. "Whither monetary economics?" International Economic Review 42, 2001, 847-870.

[25] Wallace, Neil. "General Features of Monetary Models and Their Significance." Unpublished manuscript, 2002. 